\section{Concentração de hemoglobina em crianças do nascimento até um ano de vida}

\author{
Hemoglobin concentration in children \\ from birth to one year of age
}

\author{
Sophia Cornbluth Szarfarc 1 \\ Sonia Buongermino de Souza 1 \\ Rosemeire Aparecida Victória Furumoto 2 \\ Gisela Soares Brunken 3 \\ Ana Marlúcia Oliveira Assis 4 \\ Edileuza Nunes Gaudenzi 4 \\ Rita de Cássia Ribeiro Silva 4 \\ José Maria Pacheco de Souza 5
}

\title{
Introdução
}

1 Departamento de Nutrição, Faculdade de Saúde Pública, Universidade de São Paulo, São Paulo, Brasil.

2 Departamento de Nutrição, Faculdade de Ciências da Saúde, Universidade Nacional de Brasília, Brasília, Brasil.

3 Instituto de Saúde Coletiva Universidade Federal de Mato Grosso, Cuiabá, Brasil.

4 Escola de Nutrição,

Universidade Federal da Bahia,Salvador, Brasil.

5 Departamento

de Epidemiologia,

Faculdade de Saúde Pública,

Universidade de São Paulo,

São Paulo, Brasil.

Correspondência

Sonia Buongermino de Souza

Departamento de Nutrição,

Faculdade de Saúde Pública,

Universidade de São Paulo.

Av. Dr. Arnaldo 715,

São Paulo, SP

01246-904, Brasil.

buonger@usp.br

\section{Abstract}

This study aimed to describe the evolution of hemoglobin concentration considering the following factors: birth weight, growth rate, and food intake. The cross-sectional study focused on infants (<1 year of age) whose growth and development were monitored by public health services in cities located in the five geographic regions of Brazil. Some $51.7 \%$ of the children aged 6 to 12 months presented anemia. Estimation of $[\mathrm{Hb}]$ concentration by a theoretical equation suggested that endogenous iron is able to maintain normal Hb levels in the first three months of life. Prevalence of reduced $\mathrm{Hb}$ was higher in low birth weight infants. Growth rate, verified by the difference between present weight and birth weight in $Z$ scores, was not the relevant factor for hemoglobin concentration. The relevance of breastfeeding was evident in the initial months of life. However, complementary foods did not influence infant $\mathrm{Hb}$ concentration. The high prevalence of low $\mathrm{Hb}$ levels calls attention to the need for programs to control anemia.

Anemia; Hemoglobins; Feed
A medida da concentração de hemoglobina [Hb] é, possivelmente, o indicador bioquímico mais utilizado em saúde pública, visto que ela define a deficiência nutricional de maior prevalência nos países em desenvolvimento que é a anemia.

Como tem sido constantemente referido, não estão ainda disponíveis critérios bioquímicos que permitam o diagnóstico da anemia com sensibilidade e especificidade que se apliquem a todas as idades, estados fisiológicos e gênero. O mesmo ocorre para identificação da deficiência de ferro, percursora da anemia. Indicadores bioquímicos da situação orgânica de ferro, como a concentração de ferritina e a saturação da transferrina, além de onerosos, são dependentes de equipamentos sofisticados e capacitação técnica para sua dosagem. Tampouco existem, até o momento, valores críticos específicos para caracterização de vulnerabilidade nutricional, especialmente para as crianças de baixa idade. As dificuldades apresentadas são ainda mais contundentes para o primeiro ano de vida. Valores de referência para a deficiência de ferro e/ou anemia para os lactentes no primeiro semestre de vida não estão disponíveis e o valor $11,0 \mathrm{~g} / \mathrm{dL}$, proposto para os demais períodos de vida pela Organização Mundial da Saúde (OMS) desde a década de 601 , nem sempre se mostra adequado. No entan- 
to, a deficiência de ferro, nesse período da vida, é extremamente importante, tanto que a OMS 2 relata que, mesmo moderada e na ausência de anemia, essa deficiência representa um considerável agravo à saúde, por ser esse período de intenso crescimento e diferenciação das células cerebrais, podendo ocasionar alterações de comportamento, prejuízos à capacidade motora e de aprendizagem e ainda prejudicar o crescimento 3,4,5,6. O ferro é fundamental para o funcionamento apropriado de diversas enzimas e sua deficiência pode afetar inúmeras funções metabólicas entre as quais a resposta imunológica 7 .

Os fatores que determinam a deficiência de ferro na infância estão relacionados, basicamente, à velocidade de crescimento e às reservas do mineral ao nascer. Essa reserva que foi acumulada na vida intra-uterina é proporcional ao peso e ao nascimento 8 e se esgota ao redor do quarto mês de vida, dependendo da velocidade de crescimento e do fornecimento de ferro exógeno por meio da alimentação. A tendência a um crescimento acelerado que é observado, sobretudo nas crianças nascidas com baixo peso, que têm um catch-up altamente positivo, pode levar a um esgotamento mais precoce e acelerado do ferro endógeno e aumento do risco de anemia por deficiência desse mineral. Por sua vez, a alimentação no primeiro ano de vida, por ser fundamentalmente láctea, é pobre em ferro e mesmo a introdução de alimentos sólidos e fontes do mineral não garante um bom aporte do nutriente, uma vez que as quantidades consumidas são extremamente pequenas.

Este trabalho foi desenvolvido com o objetivo de descrever a evolução da [Hb], em lactentes que freqüentam serviços públicos de saúde, considerando os fatores reconhecidos como determinantes da deficiência de ferro e/ou anemia: peso ao nascer, velocidade de crescimento e alimentação.

\section{Metodologia}

O presente estudo faz parte de um projeto mais amplo intitulado Aleitamento Materno, Dieta de Desmame e Anemia no Primeiro Ano de Vida, em Crianças Atendidas na Rede Pública de Saúde. É uma pesquisa transversal, envolvendo 5.146 crianças com idades menores de um ano, realizada em dez cidades localizadas nas cinco regiões geográficas brasileiras. Em cada localidade foram escolhidos serviços de saúde com infra-estrutura para o desenvolvimento da pesquisa. Em cada serviço, trabalhou-se com a demanda das crianças para consultas no programa de acompanhamento do crescimento e do desenvolvimento e para vacinas, obtendose, portanto, uma amostra de conveniência. Esses serviços são prioritariamente freqüentados por população de baixa renda e sem acesso a outro atendimento de saúde.

Foi estabelecido um mínimo de 500 crianças por região e, considerando a maior demanda de crianças no primeiro semestre de vida, quando se concentra a vacinação compulsória e também o maior número de consultas no programa de crescimento e desenvolvimento, fixou-se em 75 o menor número de crianças por trimestre de idade, para cada serviço de saúde.

As crianças eram incluídas no estudo após a leitura e assinatura, pelos responsáveis, de termo de consentimento esclarecido para participação.

Um formulário de respostas exclusivamente sim/não relativas à alimentação láctea e complementar foi aplicado às mães, visando identificar a prática alimentar no momento da entrevista. Os dados referentes à data de nascimento, ao peso e ao comprimento ao nascer, foram obtidos da ficha de matrícula da criança; já o peso e o comprimento atuais foram medidos no momento da entrevista.

A análise da concentração de $\mathrm{Hb}$ foi feita em sangue capilar colhido por puntura do calcanhar, utilizando-se fotômetro portátil Hemocue, recomendado pela OMS para análise de hemoglobina em estudos epidemiológicos 9 . O resultado da medida era imediatamente comunicado à mãe e ao serviço de saúde que se encarregava de orientar e intervir, sempre que necessário.

A variável idade, em meses, foi calculada dividindo os dias de vida (data da entrevista subtraída da data de nascimento) por 30,41, sendo que o valor zero corresponde às crianças com idade inferior a 16 dias.

As crianças foram classificadas em três categorias segundo o peso ao nascer; < 2.500g; $2.500<3.000 \mathrm{~g}$ e $3.000 \mathrm{~g}$ ou mais.

A situação antropométrica dos lactentes foi obtida pelo valor do escore $\mathrm{Z}$ do peso atual em relação ao padrão do National Center for Health Statistics (NCHS) 10 e a velocidade de crescimento pela diferença entre o escore $Z$ do peso atual da criança e o valor do escore $\mathrm{Z}$ do peso ao nascer. Para comparação dos resultados foram cria- 
das três categorias: crescimento relativo inferior ao esperado ( $\Delta$ escore $Z<-1$ ); crescimento relativo esperado ( $\Delta$ escore $Z$ entre $0 \pm 1$ ); e crescimento relativo acima do esperado $(\Delta$ escore $Z>+1)$.

$O$ estado nutricional das crianças foi analisado utilizando-se o índice peso/idade expresso em unidades de desvio-padrão (escore Z). Foram consideradas desnutridas crianças com escore $\mathrm{Z}$ menor do que -2, eutróficas aquelas entre $0+-2$ e com sobrepeso acima de +2 .

Quanto à alimentação, as crianças foram distribuídas em três grupos, de acordo com o tipo de leite que estavam consumindo no momento da entrevista, a saber: leite materno: quando só recebiam leite de peito; leite misto: quando recebiam leite materno e outro tipo de leite; outro leite: quando só recebiam outro tipo de leite.

Foram distribuídas, ainda, em quatro grupos, de acordo com o consumo de carnes e feijão, alimentos importantes na prevenção da anemia, visto serem fontes de ferro: sem feijão e sem carnes; sem feijão e com carnes; com feijão e sem carnes; com feijão e com carnes.

Para descrever a concentração de $\mathrm{Hb}$ foram elaboradas curvas segundo idade, peso ao nascer e velocidade de crescimento.

A equação teórica aplicada para estimar a $\mathrm{Hb}$ esperada nos primeiros 6 meses de vida, atendeu às seguintes premissas: o recém-nascido tem $75 \mathrm{mg} \mathrm{Fe} / \mathrm{kg}$ de peso, $85 \%$ dos quais sob a forma de hemoglobina 8; o volume sangüíneo corresponde a $85 \mathrm{~mL} / \mathrm{kg}$ de peso 11 e cada grama de hemoglobina contém 3,4 mg de ferro 12 .

$\mathrm{Hb}$ estimada $=(\mathrm{PN} \times 75 \times 0,85 \times 100) /(3,4 \times$ $85 \times \mathrm{PA}) \mathrm{g} / \mathrm{dL}$

onde PN e PA são, respectivamente, peso ao nascer e peso atual em $\mathrm{kg}$.

Essa equação foi aplicada para uma população padronizada cujo peso corresponde ao do percentil 50 do NCHS, e que apresenta um crescimento ideal. Esses valores foram comparados com os estimados para a amostra de estudo, e com os valores de $\mathrm{Hb}$ medidos.

A associação entre presença de valores de $\mathrm{Hb}<11 \mathrm{~g} / \mathrm{dL}$, indicativos de anemia, e as variáveis peso ao nascer, estado nutricional, tipo de leite consumido e consumo de alimentos fontes de ferro (carnes e feijão), foi analisada utilizando-se teste qui-quadrado e teste qui- quadrado de tendência. Para as análises estatísticas, usou-se o pacote estatístico Epi Info versão 6.04. Para elaboração das curvas, utilizouse o pacote estatístico STATA versão 8.0.

O presente trabalho foi aprovado pelo Comitê de Ética em pesquisa, de acordo com a resolução 196 de 10/10/1996 do Conselho Nacional de Saúde.

\section{Resultados}

A Figura 1 apresenta a curva de distribuição dos valores médios de concentração de hemoglobina da amostra. Observa-se declínio acentuado até dois meses de idade, atingindo o valor médio de hemoglobina de 10,8g/dL. A partir dos sete meses ocorre novo declínio, pouco acentuado, atingindo a concentração média de 10,3g/dL aos doze meses.

Consoante o critério da OMS 1, 55,6\% das crianças apresentaram $\mathrm{Hb}$ inferior a $11,0 \mathrm{~g} / \mathrm{dL}$, dentre esses, $51,7 \%$ tinham 6 ou mais meses de idade.

A Figura 2 mostra as curvas de concentração de hemoglobina estimada para a população padrão, estimada para a amostra e a medida. Verifica-se uma perfeita superposição das duas curvas de valores estimados.

A Figura 3 apresenta as concentrações médias de hemoglobina da amostra distribuídas segundo idade e peso ao nascer. Nota-se que as concentrações médias mais baixas ocorrem entre as crianças com peso de nascimento menor de $2.500 \mathrm{~g}$.

A Figura 4 mostra as curvas de concentração de hemoglobina segundo a velocidade de crescimento das crianças. Verifica-se que a curva das crianças com menor velocidade de crescimento apresenta valores de $\mathrm{Hb}$ maiores que os outros dois grupos. A partir de seis meses as curvas praticamente se sobrepõem.

A Tabela 1 apresenta a proporção de crianças com níveis de $\mathrm{Hb}$ abaixo de $11 \mathrm{~g} / \mathrm{dL}$, distribuídas segundo as variáveis peso ao nascer, estado nutricional, tipo de leite e consumo de alimentos fontes de ferro. Verificou-se associação estatisticamente significante entre baixo peso ao nascer e anemia $\left(\chi^{2} 2 \mathrm{gl}=79,46 ; \mathrm{p}=0,000\right)$. Foi calculado o qui-quadrado de tendência $\left(\chi^{2}\right.$ 1gl tend $=76,78 ; \mathrm{p}=0,000$ ) que mostrou que a freqüência da anemia diminui à medida que o peso ao nascer aumenta. Em relação ao estado nutricional, verificou-se que desnutrição está associada à maior prevalência de anemia $\left(\chi^{2}\right.$ $2 \mathrm{gl}=10,92 ; \mathrm{p}=0,004)$. Foi verificada a relação entre as categorias eutrófico e obeso, revelando que não existe diferença entre ambas $\left(\chi^{2} 1 \mathrm{gl}=\right.$ $0,03 ; p=0,872)$. Em seguida testou-se a relação entre desnutrido e eutrófico mais obeso $\left(\chi^{2} 1 \mathrm{gl}=\right.$ 10,89; $\mathrm{p}=0,000$ ), reforçando a associação entre desnutrição e anemia. Em relação ao tipo de leite consumido, verificou-se que a melhor situação em relação à anemia é a das crianças com leite materno seguidas por aquelas com leite misto e outro leite $\left(\chi^{2} 2 \mathrm{gl}=118,64 ; \mathrm{p}=\right.$ 0,000 ). Nota-se uma tendência ascendente na freqüência da anemia a partir da categoria com 
Concentração média de hemoglobina segundo idade em crianças no primeiro ano de vida.

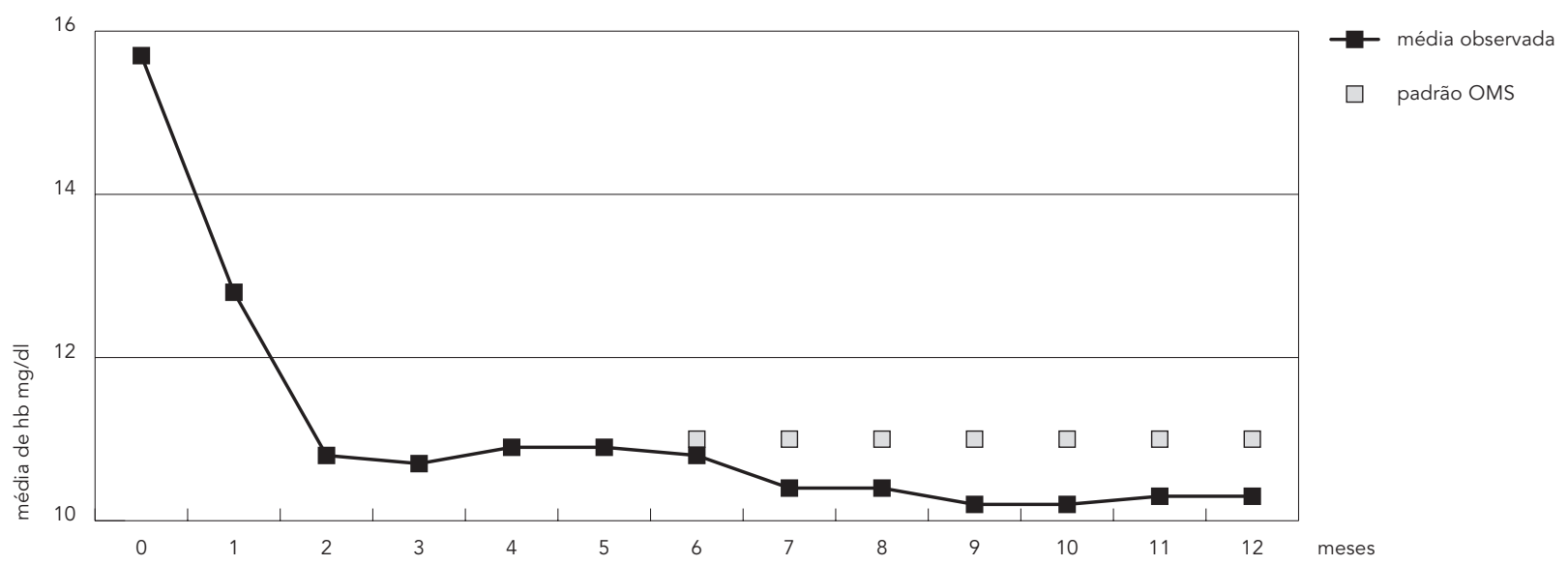

Figura 2

Concentração de hemoglobina estimada na amostra, no p50 NCHS e medida em crianças no primeiro ano de vida.

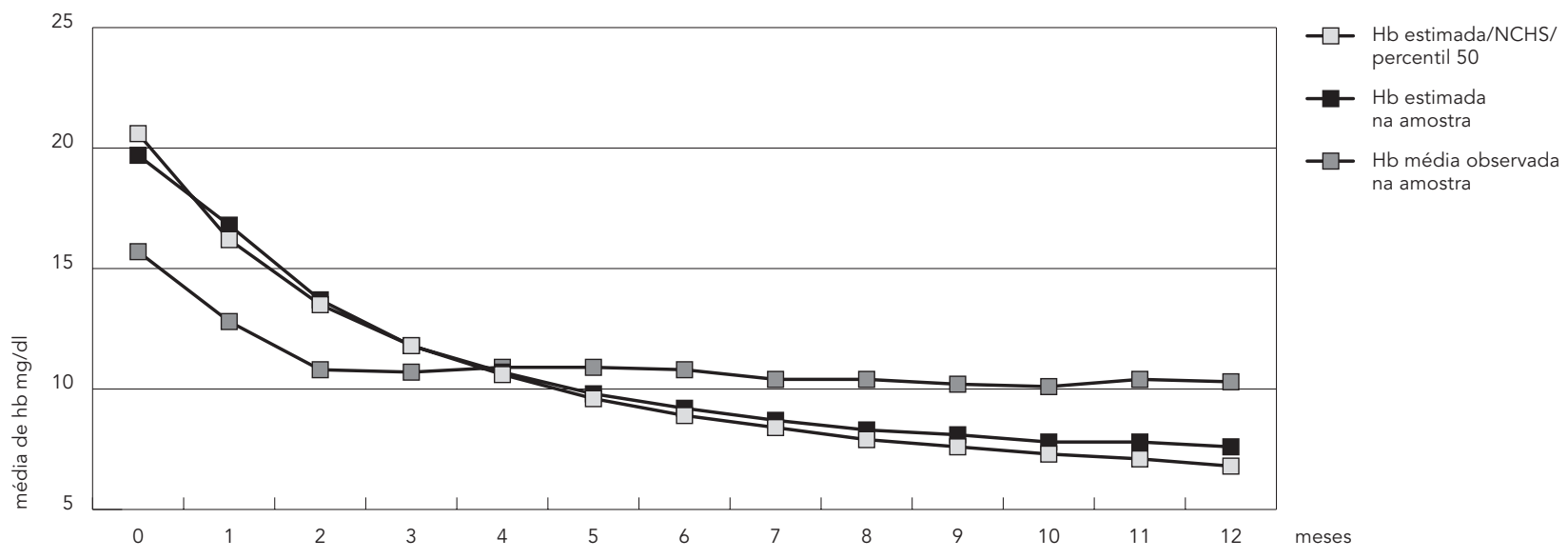


Figura 3

Concentração de hemoglobina segundo peso ao nascer e idade em crianças no primeiro ano de vida.

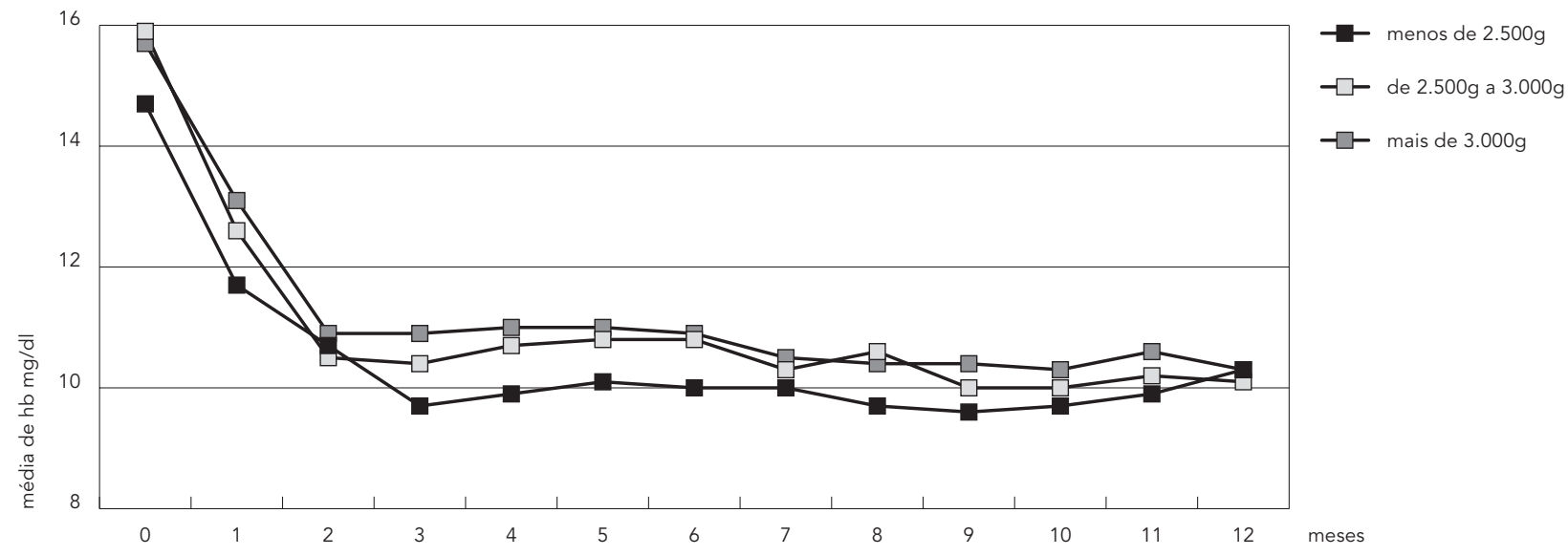

Figura 4

Concentração de hemoglobina segundo velocidade de crescimento em crianças no primeiro ano de vida.

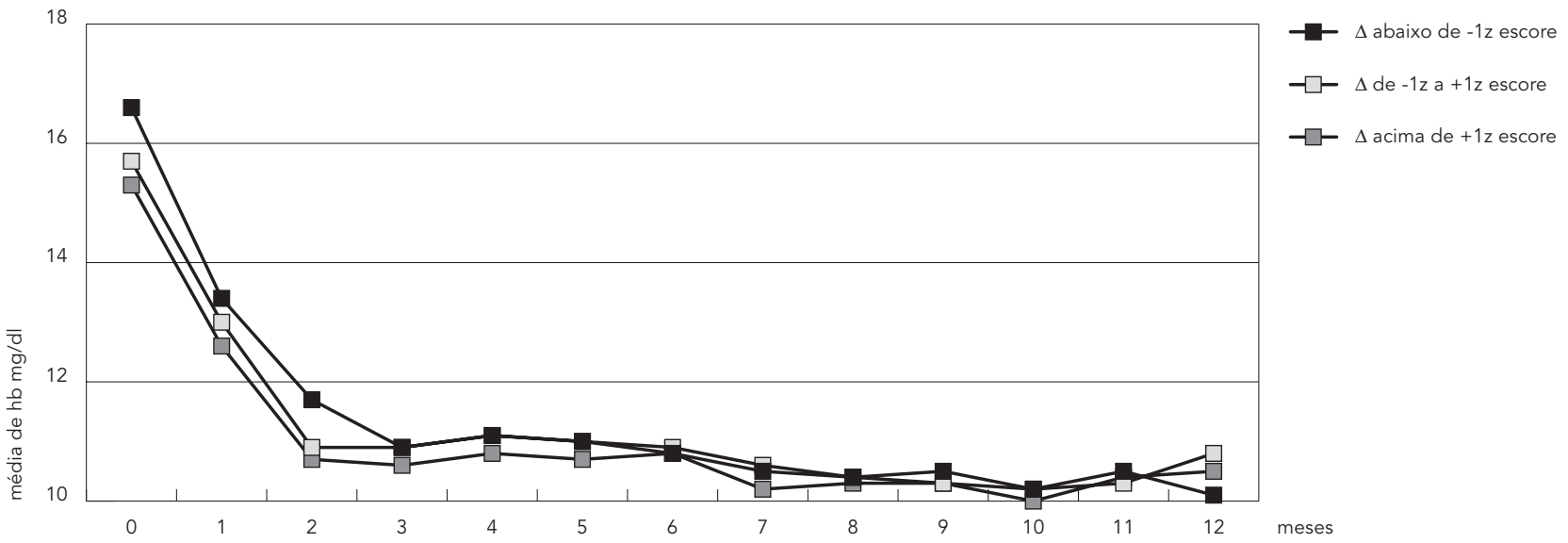


leite materno para a categoria com outro leite ( $\chi^{2}$ 1gl tend $\left.=105,88 ; \mathrm{p}=0,000\right)$. Quanto ao consumo de alimentos fontes de ferro, não foi observada diferença entre as categorias s/feijão c/carne; c/feijão s/carne; c/feijão c/carne em relação à anemia ( $\left.\chi^{2} 2 \mathrm{gl}=3,02 ; \mathrm{p}=0,221\right)$. Existe associação entre menor freqüência de anemia e a categoria s/feijão, s/carne $\left(\chi^{2} 3 \mathrm{gl}=659,71\right.$; $\mathrm{p}=0,000)$. Essa associação é verificada, também, quando se testa essa categoria contra as demais $\left(\chi^{2} 1 \mathrm{gl}=656,91 ; \mathrm{p}=0,000\right)$.

\section{Discussão}

É necessário destacar que é muito difícil o estabelecimento de valores críticos de hemoglobina como ponto de corte de normalidade entre crianças menores de 6 meses de vida, dadas as rápidas mudanças de concentração desse indicador bioquímico. Porém, considerando que a alimentação nesse período é eminentemente láctea e, portanto, carente de ferro exógeno, pode-se pressupor que os estoques endógenos do mineral são suficientes para manter a concentração de $\mathrm{Hb}$ com valores iguais ou superiores a $11,0 \mathrm{~g} / \mathrm{dL}$, valor aceito na prática clínica como referência para diagnóstico de anemia também para este grupo populacional 13, ressaltando-se, no entanto, o risco de estar subestimando a deficiência. Os estudos realizados por Saarinen et al. 14 e aqueles descritos pelo International Nutritional Anemia Consultative Group (INACG) 8 registraram, respectivamente, os valores mínimos de hemoglobina de $11,2 \mathrm{~g} / \mathrm{dL}$ e $10,5 \mathrm{~g} / \mathrm{dL}$ ao redor do segundo mês de vida. Na população deste estudo foi registrado, nessa idade, o valor médio de 10,8g/dL.

Alguns trabalhos disponíveis na literatura utilizam diferentes pontos de corte para diagnosticar a deficiência de ferro em crianças menores de seis meses: Siimes et al. 15 propõem como referência para anemia o valor $10,5 \mathrm{~g} / \mathrm{dL}$; Emond et al. 16, com base em pesquisa em que o sangue utilizado foi o capilar, como neste estudo, referem o valor 9,7g/dL. Levando-se em conta o valor de $11,0 \mathrm{~g} / \mathrm{dL}$, constatou-se que $55,6 \%$ de todas as crianças apresentaram valores de concentração de hemoglobina inferiores. Esse valor é similar ao observado entre a população de crianças do Município de São Paulo por Monteiro \& Szarfarc 17, em 1984/85, quando foram encontrados $58,1 \%$ de anêmicos. Estudo posterior, porém, mostra o aumento da prevalência dessa patologia entre lactentes paulistanos 18 .

Neste estudo, chama a atenção a grande prevalência de valores baixos de hemoglobina

\begin{tabular}{|c|c|c|c|c|}
\hline \multicolumn{5}{|c|}{$\begin{array}{l}\text { Distribuição das crianças com } \mathrm{Hb} \text { inferiores a } 11 \mathrm{~g} / \mathrm{dL} \text { segundo peso ao nascer, } \\
\text { estado nutricional, tipo de leite ingerido e ingestão de alimentos fontes de ferro. }\end{array}$} \\
\hline & \multicolumn{2}{|c|}{$[\mathrm{Hb}] \mathrm{g} / \mathrm{dL}<11.0$} & \multirow{2}{*}{$\begin{array}{c}\text { Total } \\
\mathrm{n}\end{array}$} & \multirow[t]{2}{*}{$\chi^{2}$} \\
\hline & $\mathrm{n}$ & $\%$ & & \\
\hline \multicolumn{5}{|l|}{ Peso ao nascer* } \\
\hline$<2.500 \mathrm{~g}$ & 325 & 72,9 & 446 & $\chi^{2}: 79,46$ \\
\hline $2.500-3.000 \mathrm{~g}$ & 668 & 59,1 & 1.130 & $p: 0,000$ \\
\hline $3.000 \mathrm{~g} \mathrm{e}+$ & 1.679 & 51,7 & 3.248 & $\begin{array}{c}\chi^{2} \text { tend: } 76,78 \\
\text { p: } 0,000\end{array}$ \\
\hline \multicolumn{5}{|l|}{ Estado nutricional (\%) } \\
\hline Desnutrido & 97 & 69,3 & 140 & $\chi^{2}: 10,92$ \\
\hline Eutrófico & 2.634 & 55,2 & 4.771 & $p: 0,004$ \\
\hline Obeso & 131 & 55,7 & 235 & \\
\hline \multicolumn{5}{|l|}{ Tipo de leite (\%) } \\
\hline Materno & 901 & 45,8 & 1.968 & $\chi^{2}: 118,64$ \\
\hline Misto & 891 & 59,6 & 1.495 & $\mathrm{p}: 0,000$ \\
\hline Artificial & 1.052 & 62,5 & 1.683 & $\begin{array}{c}\chi^{2} \text { tend: } 105,88 \\
\text { p:0,000 }\end{array}$ \\
\hline \multicolumn{5}{|l|}{ Fontes de ferro (\%) } \\
\hline Sem feijão; sem carnes & 901 & 49,3 & 3.131 & $\chi^{2}: 659,71$ \\
\hline Sem feijão; com carnes & 49 & 67,1 & 73 & $p: 0,000$ \\
\hline Com feijão; sem carnes & 614 & 63,1 & 973 & \\
\hline Com feijão; com carnes & 647 & 66,8 & 969 & \\
\hline Total & & & 5.146 & \\
\hline
\end{tabular}

* sem informação - 322 crianças.

entre crianças menores de 6 meses (Figura 1). Esses valores são sistematicamente mais baixos do que os observados por Brault-Dubuc et al. 19 em crianças canadenses. Devem ser destacadas, entretanto, as importantes diferenças sócio-econômicas entre as duas populações amostradas, além da alimentar, uma vez que as crianças brasileiras que freqüentam serviços públicos de saúde não recebem alimentos fortificados com ferro, o que ocorre na dieta das canadenses. $\mathrm{O}$ aumento de Hb que ocorre já a partir do segundo mês de vida verificado pelo INACG 8 , por Brault-Dubuc et al. 19 e por Monteiro \& Szarfarc 17, foi sempre maior do que o observado neste estudo.

Na tentativa de identificar por quanto tempo o ferro endógeno supre a demanda do mineral, aplicou-se uma equação teórica estabelecida, considerando que a quantidade de ferro necessária nos primeiros seis meses de vida é pequena em relação à quantidade endógena que deve ter sido armazenada na vida intrauterina. Esse ferro é redistribuído à proporção que a criança cresce; contudo, sua quantidade é mantida praticamente a mesma, visto que no 
primeiro ano de vida a dieta é eminentemente láctea e que o leite, independente da origem, é muito pobre em ferro.

Na Figura 2, que apresenta valores estimados de $\mathrm{Hb}$ considerando exclusivamente o ferro endógeno acumulado durante a vida intrauterina, verifica-se que as concentrações medidas na amostra se mantêm abaixo das curvas com valores estimados, até aproximadamente os quatro meses. Pode-se supor que a idade em que a curva real cruza a curva estimada seja a idade em que o ferro exógeno se torna indispensável para a manutenção normal da $\mathrm{Hb}$ e que, a partir dessa idade, o pouco ferro veiculado pela dieta é aproveitado de forma muito eficiente, quanto maior for sua quantidade e melhor sua qualidade. Aceita essa suposição, os valores estimados de hemoglobina até os 4 meses de idade podem ser utilizados como valores mínimos aceitáveis do indicador bioquímico, e a partir dessa idade o valor encontrado na intersecção, 11,0g Hb/dL, poderia ser utilizado como valor mínimo aceitável.

Com relação à distribuição da $\mathrm{Hb}$ segundo peso ao nascer (Figura 3), acredita-se que, possivelmente, a deficiência de ferro intra-uterina, que normalmente acomete a criança com baixo peso ao nascer (BPN), não é corrigida no primeiro ano de vida. Durante esse período, a criança apresenta valores médios de $\mathrm{Hb}$ inferiores aos encontrados para os outros grupos que mantêm entre si uma diferença mais discreta do que a observada para as crianças com BPN. Acrescenta-se, ainda, que nos serviços de saúde, onde a recomendação de prescrição de suplemento de ferro para os nascidos de baixo peso faz parte do programa de acompanhamento do crescimento e do desenvolvimento, não foi registrada a distribuição e o uso de tal composto. Sendo reconhecida a associação entre baixo peso ao nascer e níveis inadequados de hemoglobina, esse grupo populacional, sem o suplemento marcial, certamente terá um risco aumentado para as conseqüências deletérias da deficiência nutricional de ferro.

Divergindo do relatado na literatura, que coloca a velocidade de crescimento como um dos determinantes principais da deficiência de ferro nos primeiros anos de vida, a diferença dos valores de $\mathrm{Hb}$ das crianças classificadas nas três categorias de velocidade de crescimento é discreta e restrita aos primeiros meses de vida (Figura 4). Verifica-se que quanto menor a velocidade relativa de crescimento, melhor a situação em relação à $\mathrm{Hb}$, ou seja, o ferro acumulado durante o período intra-uterino é redistribuído em volumes relativos menores de sangue, permitindo manter a criança com me- nor risco de deficiência marcial. Esta é uma situação indesejável, já que a proteção à deficiência de ferro é obtida à custa de um crescimento com velocidade inferior à adequada.

Os resultados apresentados na Tabela 1 mostram que dentre os fatores aceitos como determinantes da anemia por deficiência de ferro, o peso ao nascer e o estado nutricional da criança são variáveis que se destacam. Isso remete à importância da educação nutricional no atendimento pré-natal, dando suporte para minimizar e/ou controlar o risco de baixo peso ao nascer causado pela desnutrição materna.

Em relação ao tipo de leite, verifica-se que crianças que recebem leite materno têm menor prevalência de anemia. Deve ser considerado, entretanto, que a quantidade de ferro do leite materno é muito pequena e varia durante o período de amamentação. Siimes et al. 15, num estudo longitudinal que avaliou a concentração de ferro do leite materno, verificaram que os valores declinaram de $0,56 \mathrm{mg} / \mathrm{L}$, na segunda semana de lactação, até $0,3 \mathrm{mg} / \mathrm{L}$ depois de 5 meses de lactação. Isso significa que ao mesmo tempo em que a demanda absoluta de ferro aumenta e o ferro endógeno está sendo esgotado, a quantidade do mineral veiculada pelo leite materno diminui, aumentando, sem a complementação alimentar e/ou suplementar adequada, o risco do estabelecimento da deficiência marcial.

Se por um lado os resultados deste estudo sugerem que o leite materno protege a criança da deficiência de ferro, por outro, não pode ser esquecido que este é o regime alimentar de quase a totalidade da população estudada nos três primeiros meses de vida, época em que os valores de $\mathrm{Hb}$ são elevados por conta da $\mathrm{Hb}$ fetal. À medida que a reserva de ferro diminui, diminui também a proporção de lactentes que têm o leite materno como único alimento lácteo. Possivelmente, a grande eficiência do ferro do leite materno decorra do aumento da concentração da transferrina 20 , proteína veiculadora do mineral, que propiciaria uma reciclagem muito eficiente do nutriente.

Embora com essa eficiência, valores inferiores aos aceitos como adequados foram referidos por Chaves 21 que verificou, entre crianças atendidas em Centro de Lactação, de referência para os países de língua portuguesa, concentrações médias de $\mathrm{Hb}$ de 10,0g/dL e 10,4g/dL, respectivamente aos 3 e 6 meses. Calvo et al. 22 encontraram entre crianças argentinas, alimentadas exclusivamente com leite materno, concentrações médias de $\mathrm{Hb}$ de $11,1 \mathrm{~g} / \mathrm{dL}$ aos 3 meses e 11,2g Hb/dL aos 6 meses e concluíram que, mesmo com a elevada biodisponibilidade 
de ferro do leite materno, ele é insuficiente para atender à demanda desse mineral, de uma criança com velocidade de crescimento adequado, a partir do quarto mês de vida.

Giugliani \& Victora 23 destacam a necessidade de complementação do ferro com alimentos ricos nesse micronutriente, após o sexto mês de vida, diante do balanço negativo do mineral em crianças amamentadas exclusivamente ao seio. No entanto, foi entre as crianças que consumiram rotineiramente esses alimentos (Tabela 1) que foi detectada a maior proporção de $\mathrm{Hb}$ inferior a 11,0g/dL, povavelmente em decorrência do esgotamento total da reserva de $\mathrm{Hb}$ fetal concomitante com a manutenção da criança em regime alimentar predominantemente lácteo e, portanto, pobre em ferro. Verificou-se neste estudo que os alimentos complementares fontes de ferro, feijão e carnes, são introduzidos na dieta no início do segundo semestre de vida, mas, pela pequena quantidade ingerida, é possível não interferirem no valor da $\mathrm{Hb}$. A proporção de crianças que consumia esses alimentos no primeiro semestre é mínima: 2,5\% entre menores de 5 meses e $33 \%$ entre aqueles com 5 e 6 meses de idade. Entre as crianças com mais de 6 meses, as carnes faziam parte da alimentação de $80 \%$ do grupo, enquanto o feijão participava de cerca de $40 \%$ das refeições.

Os resultados encontrados permitem concluir que o peso ao nascer é fator de relevância na determinação da deficiência de ferro, seguido da alimentação eminentemente láctea após seis meses de vida. Sendo assim, compete aos profissionais de saúde, responsáveis pelo atendimento à gestante e àqueles responsáveis pe- lo acompanhamento do crescimento e do desenvolvimento da criança, a orientação nutricional adequada para o binômio mãe/filho. Os problemas decorrentes da desnutrição na gestação e aqueles decorrentes da introdução precoce de leite não materno e/ou inadequada quanto a alimentos sólidos devem ser explicados à mãe assim como deve-se ressaltar a importância do aleitamento materno no primeiro ano de vida orientando, ao mesmo tempo, sobre a introdução correta dos alimentos complementares. Há que destacar que a introdução precoce de alimentos complementares pode ser acompanhada de contaminação, diarréia, alergia alimentar, obesidade, anemia, interferindo nas vantagens do leite materno. Por sua vez, a introdução tardia pode levar a um baixo ganho de peso e de estatura, alem de dificuldades adaptativas à alimentação da família.

Não se deve restringir a inestimável importância do aleitamento materno exclusivo (ou predominante), nos primeiros meses de vida, ao papel que representa no fornecimento de ferro biodisponível. A importância dessa prática é tal, que mesmo sendo modesta sua contribuição no que diz respeito ao mineral em pauta, o maior esforço deve ser feito pelos profissionais de saúde para manter e prolongar o tempo de aleitamento natural.

Quanto aos alimentos complementares fontes de ferro, como verificado, eles não modificam a $\mathrm{Hb}$ para as crianças do grupo etário estudado. A elevada prevalência com que ocorrem níveis baixos de hemoglobina, nessa fase da vida, no entanto, indicam a necessidade de programas de intervenção para seu controle.

\section{Resumo}

Este estudo foi desenvolvido com o objetivo de descrever a evolução da concentração de hemoglobina [Hb], considerando os fatores: peso ao nascer; velocidade relativa de crescimento e alimentação. É um estudo transversal realizado com crianças no primeiro ano de vida, atendidas para acompanhamento do crescimento e do desenvolvimento em serviços públicos de saúde, em cidades localizadas nas cinco regiões geográficas brasileiras. Verificou-se que $51,7 \%$ das crianças entre 6 e 12 meses de idade eram anêmicas. A estimativa da Hb, por meio de uma equação teórica, sugeriu que o ferro endógeno permite manter os níveis normais de Hb no primeiro trimestre de vida. A prevalência de baixa $\mathrm{Hb}$ foi maior entre as crianças com baixo peso ao nascer. A velocidade relativa de crescimento, avaliada pela diferença do peso atual e peso ao nascer, em unidades de $Z$ escore, não foi fator de relevância para a Hb. A importância do aleitamento materno foi evidente nos primeiros meses de vida. Verificou-se,entretanto, que os alimentos complementares não influenciaram a Hb. A elevada prevalência de níveis baixos de hemoglobina indica a necessidade de enfatizar programas de intervenção para o controle da anemia ferropriva.

Anemia; Hemoglobina; Alimentação 


\section{Colaboradores}

S. C. Szarfarc e S. B. Souza foram responsáveis pelo treinamento e qualidade dos dados e pela elaboração do artigo. J. M. P. Souza responsabilizou-se pelas análises estatísticas. Os demais co-autores participaram de todas as fases da elaboração tanto da coleta/colheita de dados como da análise dos mesmos e elaboração do texto do artigo.

\section{Referências}

1. Organização Mundial da Saúde. Grupo Científico de Anemias Nutricionales. Serie de Informes Técnicos 405. Genebra: Organização Mundial da Saúde; 1968.

2. Organización Mundial de la Salud. Lucha contra la anemia nutricional, especialmente contra la carencia de hierro. Serie de Informes Técnicos 580. Geneva: Organización Mundial de la Salud; 1975.

3. Demaeyer E. Preventing and controlling iron deficiency anaemia through primary health care: a guide for health administrators and programme managers. Geneva: World Health Organization; 1989.

4. Walter T, De Andraca I, Chadud P, Perales CG. Iron deficiency anemia: adverse effects on infant psychomotor development. Pediatrics 1989; 84:717.

5. Lozoff B, Jimenez E, Hagen J, Mollen E, Wolf AW. Poor behavioral and development outcome more than 10 years after treatment for iron deficiency in infancy. Pediatrics 2000; 105:1-4.

6. Saboojee H, Pettifor JM. Iron deficiency and impaired child development. Br Med J 2001; 323: 1377-78.

7. Blum M. Fortificcion de alimentos. Nutriview 1997; Edición Especial:1-10.

8. International Nutritional Anemia Consultative Group. Iron deficiency in infancy and childhood. Washington DC: Pan American Health Organization; 1979.

9. Thomas-Hudson M, Bingham KC, Simmons WK. A evaluation of the Hemocue for measuring haemoglobin in field studies in Jamaica. Bull World Health Organ 1994; 73:423-6.

10. National Center for Health Statistcs. Growth curves for children, birth - 18 years, United States. Vital Health Stat 1977; 11:1-74.

11. Brandalise SR, Matsuda E. Anemias carenciais. In: Nóbrega FJ, organizador. Desnutrição intrauterina e pós natal. São Paulo: Editora Panamed; 1981. p. 395-405.

12. White A, Handler P, SmithEL. Hemoproteínas e porfirinas. In: White A, Handler P, Smith EL, organizadores. Princípios de bioquímica. Rio de Janeiro: Editora Guanabara Koogan; 1976. p. 139-50.

\section{Agradecimentos}

À Fundação de Amparo à Pesquisa do Estado de São Paulo, pelo financiamento do Projeto Aleitamento Materno, Dieta de Desmame e Anemia no Primeiro Ano de Vida, em Crianças Atendidas na Rede Pública de Saúde, do qual foi elaborado este artigo.
13. Wintrobe M, Lee GR, BogsTR, Bithell TC, Foerster J, Athens J, et al. Clinical hematology. 8th Ed. Philadelphia: Lea \& Febiger; 1981.

14. Saarinen UM, Siimes MA. Developmental changes in red blood cell counts and indices of infants after exclusion of iron deficiency by laboratory criteria and continuous iron supplementation. J Pediatr 1978; 92:412-6.

15. Siimes MA, Salmenperä L, Perheentupa J. Exclusive breast-feeding for 9 months: risk of iron deficiency. J Pediatr 1984; 104:196-9.

16. Emond AM, Hawkins N, Pennock C, Golding J. Haemoglobin and ferritin concentrations in infants at 8 months of age. Arch Dis Child 1996; 74:36-9.

17. Monteiro CA, Szarfarc SC. Estudo das condições de saúde das crianças do Município de São Paulo, SP (Brasil), 1984/1985. V. Anemia. Rev Saúde Pública 1987; 21:255-60.

18. Monteiro CA, Szarfarc SC, Mondini L. Tendência secular da anemia na infância na Cidade de São Paulo. Rev Saúde Pública 2000; 34 Suppl :62-72.

19. Brault-Dubuc M, Nadeau M, Dickie J. Iron status of French-Canadian children: a three year followup study. Hum Nutr Appl Nutr 1983; 37A:210-21.

20. Cook JD, Skikne B, Baynes R. The use of the serum transferin receptor for the assessment of iron status. In: Hallberg L, George N, editors. Iron nutrition in health and disease. London: John Libbey; 1996. p. 49-58.

21. Chaves SP. Crescimento e concentração de hemoglobina de lactentes em aleitamento materno exclusivo [Tese de Doutorado]. São Paulo: Faculdade de Saúde Pública da Universidade de São Paulo; 2000.

22. Calvo EB, Galindo AC, Aspres NB. Iron status in exclusively breast-fed infants. Pediatrics 1992; 90:375-9.

23. Giugliani ERJ, Victora CG. Normas alimentares para crianças brasileiras menores de dois anos: bases científicas. Brasília: Organização Pan-Americana da Saúde; 1997.

Recebido em 4/Out/2002

Versão final reapresentada em 13/Mai/2003

Aprovado em 24/Out/2003 